\title{
All Pay Quality-Bids in Score Procurement Auctions*
}

\author{
Dan Kovenock ${ }^{\dagger} \quad$ Jingfeng $\mathrm{Lu}^{\ddagger}$
}

January 2020

\begin{abstract}
In this paper, we study score procurement auctions with all-pay quality bids. A supplier's score is the difference between his quality and price bids. The supplier with the highest score wins and gets paid his own price bid. The procurer's payoff is the difference between the winner's quality and the procurer's payments to the suppliers. Equilibrium quality and price bids are solved without first obtaining the corresponding equilibrium scores. We find that quality bids, the suppliers' payoffs and the procurer's payoff do not depend on whether price bids are made contingent on quality bids. Compared to a benchmark of winner-pay quality bids, in which the losing suppliers' quality bidding costs are reimbursed by the procurer, all-pay quality bids tend to reduce quality provision and suppliers' payoffs, but they tend to increase the total surplus and the procurer's payoff.
\end{abstract}

JEL Classification Numbers: C70, D44, D89, L12, O32.

Keywords: All-pay quality bids; Equilibrium analysis; Score auctions; Score procurements; Winner-pay quality bids.

\footnotetext{
${ }^{*}$ We are grateful to Estelle Cantillon, Yeon-Koo Che, Christian Ewerhart, Vijay Krishna, Igor Letina, Shuo Liu, Joao Montez, Sergio Parreiras, Ron Siegel and Jun Xiao for helpful comments and discussions. We thank Dongri Liu and Lirong Yang for excellent research assistance. Any remaining errors are our own.

${ }^{\dagger}$ Dan Kovenock: Economic Science Institute, Chapman University, One University Drive, Orange, CA 92866, USA. Phone: (1)714-628-7226. Email: kovenock@chapman.edu.

${ }^{\ddagger}$ Jingfeng Lu: Department of Economics, National University of Singapore, 1 Arts Link, Singapore $117570 . \quad$ Phone: (65)65166026. Email: ecsljf@nus.edu.sg.
} 


\section{Introduction}

Procurement is widely adopted to acquire goods, services, or work. On average, about $15 \%$ of yearly global domestic product is spent on public procurement alone, including military acquisitions. ${ }^{1}$ Procurements involving multi-dimensional bids are ubiquitous. Typically, suppliers are required to bid on both quality and price, which jointly form single-dimensional scores that are used by the procurer to determine the winner, whose offer turns out to be the most economically advantageous.

Since the seminal work of Che [7], score auctions with winner-pay quality bids have been studied in the literature in many contexts, including Branco [6], Asker and Cantillon [1, 2], Bajari, Houghton and Tadelis [3], Wang and Liu [14], Hanazono, Nakabayashi and Tsuruoka [9] and Nishimura [12]. In this literature, the central idea in identifying equilibrium bidding strategies is to transform the multi-dimensional bidding problem into a single-dimensional problem of bidding on score. This idea is based on the insightful observation that a supplier should choose quality and price bids optimally to maximize his payoff conditional on winning with any score $s$. The optimal quality and price choice for any score $s$ would allow the definition of a value function for each supplier, which only depends on own type and the score bid $s$. With this value function, the original problem with multi-dimensional bids is transformed into a standard single-dimensional problem with a score bid. ${ }^{2}$

In many situations, the quality bids are more realistically interpreted as an all-pay component (at least partially) rather than a winner-pay component. ${ }^{3}$ For example, all-pay quality bids are often features of procurements in defense contracting and other military design competitions, architectural designs, government construction projects with a design component, and business to business customized sales. To the best of our knowledge, Che and Gale [8] are the first to study score procurement with all-pay quality bids in a complete information environment. Their focus is to demonstrate the optimality of shortlisting and handicapping in such environments.

Our study is the first to introduce all-pay quality bids in score procurement auctions with incomplete information. We provide an alternative procedure to identify the unique symmetric pure strategy equilibrium with scores monotonic in types, if such an equilibrium exists. The procedure applies to both cases of all-pay and winner-pay quality bids. ${ }^{4}$ The merit of our alternative procedure lies in that we directly identify the quality bid and price bid without relying on an intermediate step of solving for the score bid. This procedure is made possible because of another observation on the relationship between equilibrium price and quality bids in score auctions, which has not been utilized so far in the literature: We can view the pair of equilibrium

\footnotetext{
${ }^{1}$ See "Supplement to the 2013 Annual Statistical Report on United Nations Procurement: Procurement and Innovation," https://www.unsystem.org/content/supplement-2013-annual-statistical-report-united-nations-procurement (accessed Jan. 15, 2019).

${ }^{2}$ For a recent general treatment of winner-pay quality-bid score auctions, please refer to Hanazono, Nakabayashi and Tsuruoka [9] for details.

${ }^{3}$ See Baye, Kovenock and de Vries [4] and Kaplan, Luski, Sela and Wettstein [10] among many others.

${ }^{4}$ Our procedure also applies to intermediate cases in Siegel [13].
} 
quality and price bids as an incentive compatible direct mechanism, with the quality bid being the allocation rule and the price bid the payment rule. Then, as well understood from Myerson [11], the price bidding function can be fully pinned down as a function of the quality bidding function. This observation together with the well utilized insight that a supplier chooses quality and price bids optimally to maximize his payoff conditional on bidding the equilibrium score, would allow us to obtain a condition on the equilibrium quality bidding function which, together with the appropriate boundary condition, identifies that function. It thus follows that we can further identify the equilibrium price bidding function based on the above mentioned relationship.

The equilibrium analysis enables us to study the effect of all-pay quality bids in score procurement auctions on quality provision, supplier payoffs, procurer payoffs and total surplus by comparing our environment to two benchmarks with winner-pay quality bids. In Benchmark I, we have a hypothetical scenario in which placing a quality bid does not incur sunk cost. In Benchmark II, we assume the losing suppliers' sunk costs incurred in placing their quality bids are reimbursed by the procurer. Our setting thus resembles those of Baye, Kovenock and de Vries [5] and Siegel [13] who study equilibria in auctions with contingent investment. ${ }^{5}$

Specifically, we adopt a two-supplier environment, in which a supplier's score is the difference between his quality and price bids. The supplier with the higher score wins and gets paid his own price bid. The procurer's payoff equals the difference between the winner's quality and her payments to the suppliers. We find that compared to the benchmark II environment of winner-pay quality bids, in which the losing supplier's cost of quality bid is reimbursed by the procurer, all-pay quality bids tend to reduce quality provision and the suppliers' payoffs, but they tend to increase the total surplus and procurer's payoff. Compared to the hypothetical benchmark I, in which placing a quality bid does not incur sunk cost, all pay quality bids tend to reduce quality provision, the suppliers' payoffs and total surplus, although they can either increase or decrease the procurer's payoff. Consequently, although it is reasonable to view the credible assurance of a given quality level in many procurement contexts as requiring an upfront sunk investment that is all-pay in nature, our approach suggests an additional reason for adopting all-pay quality bids in score procurement auctions: the practice may increase the procurer's payoff.

Moreover, we find that quality bids, supplier payoffs and the procurer's payoff do not depend on whether quality bids are placed and publicly revealed before the price bids are made. Our result shows that when quality and price must be placed in sequence, whether or not information on quality bids is revealed does not affect procurement performance.

The rest of paper is organized as follows. Section 2 sets up the model with all-pay quality bids. Section 3 provides a procedure for equilibrium analysis, which applies to both all-pay and winner-pay quality bids, as well as to a continuum of intermediate cases. Section 4 examines an environment in which quality bids are

\footnotetext{
${ }^{5}$ Baye, Kovenock and de Vries [5] study auctions with incomplete information, and Siegel [13] studies settings with complete information.
} 
placed and publicly revealed before price bids are made. Section 5 compares all-pay and winner-pay quality bids on quality provision, supplier payoffs, the procurer's payoff and total surplus. Section 6 provides some concluding remarks. Technical proofs are relegated to the appendices.

\section{Model}

We consider a score procurement auction with a risk neutral procurer and two risk neutral suppliers. The two suppliers, $i=1,2$, simultaneously choose their nonnegative bids of quality $q_{i}$ and price $p_{i}$ to compete for a contract. Only the winner will collect his price bid $p$, while the quality bid $q$ is an all pay component paid by each bidder. Bidder $i$ must incur a total cost of $C_{i}(q)=c_{i} \varsigma(q)$ to bid a quality $q$ whether he wins the contract or not. Here, we assume the function $\varsigma(\cdot)$ is continuous on $[0,+\infty)$, with $\varsigma(0)=\varsigma^{\prime}(0)=0, \varsigma^{\prime}(q)>0, \forall q>0$, and $\varsigma^{\prime \prime}(q)>0, \forall q \geq 0 .{ }^{6}$ For example, the class of functions $\varsigma(q)=q^{\gamma}, \gamma>1$ satisfies these conditions. $c_{i}$ is supplier $i$ 's private type, with high $c_{i}$ leading to high marginal effort cost. We assume that the $c_{i}$ are independently and identically distributed following cumulative distribution $F(\cdot)$ on $[\underline{c}, \bar{c}]$ with a positive density $f(\cdot)$. We assume $0<\underline{c}<\bar{c}<+\infty$.

The higher score wins and the score function is defined as

$$
s(q, p)=q-p
$$

The procurer's payoff is the difference between the $q$ and $p$ of the winning bidder. Supplier $i$ 's payoff is $p_{i}-C_{i}\left(q_{i}\right)$ if he wins, otherwise it is $-C_{i}\left(q_{i}\right)$.

\section{Equilibrium analysis}

We now derive the unique equilibrium within the class of symmetric pure strategy equilibria with scores decreasing in type. There are two key observations that facilitate identifying the equilibrium. Suppose the score of type $c_{i}$ is $s\left(c_{i}\right)$ in the equilibrium. First, if we view the equilibrium strategy $\left(q\left(c_{i}\right), p\left(c_{i}\right)\right)$ as an incentive compatible direct mechanism, then the Myerson [11] approach, together with a zero payoff condition for type $\bar{c}$, would allow us to pin down a payment rule $p\left(c_{i} ; q(\cdot)\right)$ for any given allocation rule $q(\cdot)$ decreasing in type. Second, define the score by $s\left(c_{i}\right)=q\left(c_{i}\right)-p\left(c_{i} ; q(\cdot)\right), \forall c_{i}$. Note $s(\cdot)$ is solely determined by the allocation rule $q(\cdot)$. Given supplier $i$ with type $c_{i}$ bids this score $s\left(c_{i}\right)$ in equilibrium, supplier $i$ would choose a pair $(q, p)=\left(q\left(c_{i}\right), p\left(c_{i}\right)\right)$ when maximizing his expected payoff. This further gives us conditions on the equilibrium bidding functions $(q(\cdot), p(\cdot))$. The restrictions on $(q(\cdot), p(\cdot))$, which are implied by the above two observations, together with the boundary condition $q(\bar{c})=0$, pins down a candidate equilibrium allocation rule $q(\cdot)$. If this $q(\cdot)$ is decreasing in type, and the corresponding $s(\cdot)$ is also decreasing in type, then

\footnotetext{
${ }^{6} \varsigma^{\prime}(0)$ and $\varsigma^{\prime \prime}(0)$ refer to the right hand derivatives at zero.
} 
the identified $(q(\cdot), p(\cdot))$ is the symmetric pure strategy equilibrium. Otherwise, there exists no equilibrium within the class.

We will demonstrate that the above two observations allow us to derive the unique symmetric pure strategy equilibrium with scores decreasing in type, ${ }^{7}$ when such an equilibrium exists, and to determine when no such equilibrium exists. To accommodate both cases of an all-pay quality bid and a winner-pay quality bid, following Baye, Kovenock and de Vries [5] and Siegel [13], we introduce a parameter $\rho \in\{0,1\}$ in the following analysis, where $\rho$ represents the fraction of the quality bid that is all-pay. ${ }^{8}$ Hence, $\rho=1$ stands for an all-pay quality bid, which is the main focus of this paper; and $\rho=0$ stands for the hypothetical benchmark scenario of a winner-pay quality bid, in which a losing supplier does not incur a cost to place his quality bid (Benchmark I). The other benchmark scenario of winner-pay quality bid we are going to study is the case, in which we have $\rho=1$ but the losing supplier's cost of placing his quality bid is reimbursed by the procurer (Benchmark II). Note that the suppliers' payoff functions are identical in these two benchmark environments, thus their equilibrium bidding strategies must be the same. Because of the transfers from the procurer to the losing suppliers, the procurer's payoff must be lower in Benchmark II.

\section{Implications of the first observation (Myerson incentive compatibility)}

We first consider the implications of the first observation. Fix any $q\left(c_{i}\right)$. We can view any equilibrium strategy $\left(q\left(c_{i}\right), p\left(c_{i}\right)\right)$ as an incentive compatible direct mechanism that leads to decreasing scores in type. The quality bid function $q\left(c_{i}\right)$ can be viewed as an allocation rule, and the price function $p\left(c_{i}\right)$ can be viewed as a payment rule. Then the Myerson approach allows us to pin down the payment rule $p\left(c_{i}\right)$ as a function of the allocation rule $q(\cdot)$. The following provides the details.

Given a $\left(q\left(c_{i}\right), p\left(c_{i}\right)\right)$ pair that leads to scores that are decreasing in type, consider a type $c_{i}$ supplier's problem of choosing a $\tilde{c}_{i}$ to maximize his expected payoff when the other supplier is truthful:

$$
\max _{\tilde{c}_{i}} \pi_{i}\left(\tilde{c}_{i}, c_{i}\right)=\left[1-F\left(\tilde{c}_{i}\right)\right]\left[p\left(\tilde{c}_{i}\right)-(1-\rho) c_{i} \varsigma\left(q\left(\tilde{c}_{i}\right)\right)\right]-\rho c_{i} \varsigma\left(q\left(\tilde{c}_{i}\right)\right)
$$

If $(q(\cdot), p(\cdot))$ is a pure strategy equilibrium, then $\tilde{c}_{i}^{*}$, the solution to (2), satisfies $\tilde{c}_{i}^{*}=c_{i}, \forall c_{i}$. In other words, the following incentive compatibility (IC) condition holds:

$$
c_{i}=\arg \max _{\tilde{c}_{i}} \pi_{i}\left(\tilde{c}_{i}, c_{i}\right)=\left[1-F\left(\tilde{c}_{i}\right)\right]\left[p\left(\tilde{c}_{i}\right)-(1-\rho) c_{i} \varsigma\left(q\left(\tilde{c}_{i}\right)\right)\right]-\rho c_{i} \varsigma\left(q\left(\tilde{c}_{i}\right)\right) .
$$

\footnotetext{
${ }^{7}$ The identified equilibrium score, quality and price bids are all differentiable in type.

${ }^{8}$ The equilibrium analysis in this section applies to an environment with a general number of suppliers, $\rho \in[0,1]$ and score function $s=\alpha q-\beta p, \alpha, \beta>0$.
} 
If $q^{\prime}\left(c_{i}\right)<0$, the single crossing condition holds:

$$
\begin{aligned}
\frac{\partial^{2} \pi_{i}\left(\tilde{c}_{i}, c_{i}\right)}{\partial \tilde{c}_{i} \partial c_{i}} & =-\frac{d\left\{\left[1-F\left(\tilde{c}_{i}\right)\right](1-\rho) \varsigma\left(q\left(\tilde{c}_{i}\right)\right)+\rho \varsigma\left(q\left(\tilde{c}_{i}\right)\right)\right\}}{d \tilde{c}_{i}} \\
& =f\left(\tilde{c}_{i}\right)(1-\rho) \varsigma\left(q\left(\tilde{c}_{i}\right)\right)-\left[1-F\left(\tilde{c}_{i}\right)\right](1-\rho) \varsigma^{\prime}\left(q\left(\tilde{c}_{i}\right)\right) q^{\prime}\left(\tilde{c}_{i}\right)-\rho \varsigma^{\prime}\left(q\left(\tilde{c}_{i}\right)\right) q^{\prime}\left(\tilde{c}_{i}\right) \\
& >0
\end{aligned}
$$

which means such $q\left(c_{i}\right)$ can be supported by the pricing rule that will be identified below.

The IC condition (3), together with the envelope theorem, thus leads to

$$
\frac{d \pi_{i}\left(c_{i}, c_{i}\right)}{d c_{i}}=-\left\{\left[1-F\left(c_{i}\right)\right](1-\rho) \varsigma\left(q\left(c_{i}\right)\right)+\rho \varsigma\left(q\left(c_{i}\right)\right)\right\} .
$$

Note that in equilibrium with scores monotonic in type, regardless of the value of $\rho$, we must have $\pi_{i}(\bar{c}, \bar{c})=0$. In equilibrium, the least efficient type $\bar{c}$ never wins and never incurs a cost of quality. ${ }^{9}$

Using the envelope condition (4), we have

$$
\pi_{i}\left(c_{i}, c_{i}\right)=\int_{c_{i}}^{\bar{c}}\{[1-F(t)](1-\rho) \varsigma(q(t))+\rho \varsigma(q(t))\} d t .
$$

By the definition of $\pi_{i}\left(\tilde{c}_{i}, c_{i}\right)$ in (2), we further have

$$
\begin{aligned}
\pi_{i}\left(c_{i}, c_{i}\right) & =\left[1-F\left(c_{i}\right)\right]\left[p\left(c_{i}\right)-(1-\rho) c_{i} \varsigma\left(q\left(c_{i}\right)\right)\right]-\rho c_{i} \varsigma\left(q\left(c_{i}\right)\right) \\
& =\int_{c_{i}}^{\bar{c}}\{[1-F(t)](1-\rho) \varsigma(q(t))+\rho \varsigma(q(t))\} d t,
\end{aligned}
$$

which leads to price rule

$$
p\left(c_{i}\right)=\frac{\int_{c_{i}}^{\bar{c}}\{[1-F(t)](1-\rho) \varsigma(q(t))+\rho \varsigma(q(t))\} d t+\rho c_{i} \varsigma\left(q\left(c_{i}\right)\right)}{1-F\left(c_{i}\right)}+(1-\rho) c_{i} \varsigma\left(q\left(c_{i}\right)\right) \geq 0 .
$$

\section{Implications of the second observation (optimal bids for a given score)}

We now turn to the implication of the second observation. Suppose $\left(q\left(c_{i}\right), p\left(c_{i}\right)\right)$ is a symmetric pure strategy equilibrium. By implication of the first observation, we must have that (6) holds. Define

$$
s\left(c_{i}\right)=s\left(q\left(c_{i}\right), p\left(c_{i}\right)\right) .
$$

The second observation says that any equilibrium $\left(q\left(c_{i}\right), p\left(c_{i}\right)\right)$ must solve the following optimization problem:

$$
\begin{aligned}
\left(q\left(c_{i}\right), p\left(c_{i}\right)\right) & =\arg \max _{(q, p)}\left\{\left[1-F\left(c_{i}\right)\right]\left[p-(1-\rho) c_{i} \varsigma(q)\right]-\rho c_{i} \varsigma(q)\right\} \\
\text { s.t. } \quad & : \quad s=q-p=s\left(c_{i}\right) .
\end{aligned}
$$

\footnotetext{
${ }^{9}$ Note $q(\bar{c})=0$ for $\rho=1$.
} 
The corresponding Lagrangian for the above problem is

$$
\max _{(q, p, \lambda)} L(q, p, \lambda)=\left[1-F\left(c_{i}\right)\right]\left[p-(1-\rho) c_{i} \varsigma(q)\right]-\rho c_{i} \varsigma(q)+\lambda\left[(q-p)-s\left(c_{i}\right)\right]
$$

The first order conditions are as follows:

$$
\begin{aligned}
& \frac{L(q, p, \lambda)}{\partial q}=-\left[1-F\left(c_{i}\right)\right](1-\rho) c_{i} \varsigma^{\prime}(q)-\rho c_{i} \varsigma^{\prime}(q)+\lambda=0, \\
& \frac{L(q, p, \lambda)}{\partial p}=\left[1-F\left(c_{i}\right)\right]-\lambda=0, \\
& \frac{L(q, p, \lambda)}{\partial \lambda}=(q-p)-s\left(c_{i}\right)=0 .
\end{aligned}
$$

We thus have

$$
\varsigma^{\prime}(q)=\frac{1-F\left(c_{i}\right)}{c_{i}\left\{\left[1-F\left(c_{i}\right)\right](1-\rho)+\rho\right\}}=\frac{1}{c_{i}\left[(1-\rho)+\frac{\rho}{1-F\left(c_{i}\right)}\right]} .
$$

We can thus pin down the candidate equilibrium quality

$$
q\left(c_{i}\right)=\left(\varsigma^{\prime}\right)^{-1}\left(\frac{1}{c_{i}\left\{(1-\rho)+\frac{\rho}{1-F\left(c_{i}\right)}\right\}}\right) .
$$

Note that the quality bid $q\left(c_{i}\right)$ is identified by only using the second observation. This is the case for the difference-form score function that we consider, but it is not the case in general. Please refer to Appendix $B$ for the procedure for identifying $q\left(c_{i}\right)$ without solving for the equilibrium score bidding function for a general score function. In particular, the case of a ratio score function is studied there as an example.

Clearly, for an all-pay quality bid with $\rho=1$, we have $q(\bar{c})=\left(\varsigma^{\prime}\right)^{-1}(0)=0 .{ }^{10}$ For a winner-pay quality bid with $\rho=0$, we have $q(\bar{c})=\left(\varsigma^{\prime}\right)^{-1}\left(\frac{1}{\bar{c}}\right)>0$ since $\varsigma^{\prime \prime}(\cdot)>0$. Clearly, we have $q^{\prime}(\cdot)<0$, since

$$
q^{\prime}\left(c_{i}\right)=\frac{\left[\frac{1}{c_{i}\left\{(1-\rho)+\frac{\rho}{1-F\left(c_{i}\right)}\right\}}\right]^{\prime}}{\varsigma^{\prime \prime}\left(q\left(c_{i}\right)\right)}<0 .
$$

Since $q\left(c_{i}\right)$ is decreasing, the above characterized $(q(\cdot), p(\cdot))$ would constitute a symmetric pure strategy equilibrium within the class we consider if and only if the corresponding score function $s\left(c_{i}\right)=q\left(c_{i}\right)-p\left(c_{i}\right)$ is decreasing in type. This is indeed the case as verified by the following lemma, whose proof is relegated to appendix $A$.

Lemma $1 s^{\prime}\left(c_{i}\right)=q^{\prime}\left(c_{i}\right)-p^{\prime}\left(c_{i}\right)<0$.

We thus have the following theorem.

Theorem 1 The symmetric bidding strategy $(q(\cdot), p(\cdot))$ identified in (9) and (6) constitutes the unique symmetric pure strategy equilibrium with scores decreasing in types. Moreover, $q(\cdot)$ is decreasing in type.

\footnotetext{
${ }^{10}$ In general, $q(\bar{c})=0$ holds for any $\rho \in(0,1]$.
} 
The way we establish the equilibrium implies that the following two remarks are straightforward.

Remark 1 The equilibrium analysis of this section applies to $\rho \in[0,1]$.

Remark 2 Our equilibrium characterization requires $\varsigma^{\prime}(0)=0$, which says marginal cost must be zero when quality is zero. Che and Gale [8] also require the same condition in their equilibrium analysis in a complete information score auction setting. If $\varsigma^{\prime}(0)>0$, the equilibrium involves pooling. For example, consider $\rho=1$. Define the cutoff $\hat{c}$ by $\varsigma^{\prime}(0)=\frac{1-F(\hat{c})}{\hat{c}}$. In equilibrium, all types in $[\hat{c}, \bar{c}]$ place the same bid as type $\bar{c} .^{11}$

We have the following two additional remarks on applying our procedure to a more general environment.

Remark 3 The above two-step procedure for identifying the symmetric pure strategy equilibrium if it exists can be generalized to an environment with an arbitrary number of suppliers and a general class of score functions $s(q, p)$. The details are included in Appendix B. In an application there with score function $s=$ $s(q, p)=\frac{q}{p}$ and $N \geq 2$ suppliers, ${ }^{12}$ we show that if $\varsigma^{\prime}(0)=0$, then a symmetric pure strategy equilibrium generating scores decreasing in type does not exist for the case of all-pay quality bids. However, if $\varsigma^{\prime}(0)>0$, then the equilibrium for the case of all-pay quality bids is identified as

$$
\begin{aligned}
& q\left(c_{i}\right)=\left(\varsigma^{\prime}\right)^{-1}\left(\frac{\bar{c} \varsigma^{\prime}(0)}{c_{i}}\right), \\
& p\left(c_{i}\right)=\frac{\int_{c_{i}}^{\bar{c}} \varsigma(q(t)) d t+c_{i} \varsigma\left(q\left(c_{i}\right)\right)}{\left[1-F\left(c_{i}\right)\right]^{N-1}} .
\end{aligned}
$$

Remark 4 Following the insights of Hanazono, Nakabayashi and Tsuruoka [9], there is an alternative approach, which instead requires identifying an equilibrium score function as an intermediate step before decomposing it into quality and price bids. The details are as fellows. ${ }^{13}$

Assume a general score rule of $s(q, p)$ and $N \geq 2$ suppliers. From the second observation, if $s\left(c_{i}\right)$ is the equilibrium score, we can solve for

$$
\begin{aligned}
\left(q\left(c_{i} \mid s\left(c_{i}\right)\right), p\left(c_{i} \mid s\left(c_{i}\right)\right)\right) & =\underset{(q, p)}{\arg \max }\left[1-F\left(c_{i}\right)\right]^{N-1}\left[p-(1-\rho) c_{i} \varsigma(q)\right]-\rho c_{i} \varsigma(q), \\
\text { s.t. } & : \quad s(q, p)=s\left(c_{i}\right) .
\end{aligned}
$$

Then consider supplier i's optimization problem

$$
\max _{\tilde{c}_{i}} \pi_{i}\left(\tilde{c}_{i}, c_{i}\right)=\left[1-F\left(\tilde{c}_{i}\right)\right]^{N-1}\left[p\left(\tilde{c}_{i} \mid s\left(\tilde{c}_{i}\right)\right)-(1-\rho) c_{i} \varsigma\left(q\left(\tilde{c}_{i} \mid s\left(\tilde{c}_{i}\right)\right)\right)\right]-\rho c_{i} \varsigma\left(q\left(\tilde{c}_{i} \mid s\left(\tilde{c}_{i}\right)\right)\right) .
$$

\footnotetext{
${ }^{11}$ This is implied by (9).

${ }^{12}$ The equilibrium analysis in the application applies to a score function of $s=s(q, p)=\frac{q^{\mu}}{p^{\tau}}$ with $\mu, \tau>0$.

${ }^{13}$ Our approach avoids this additional intermediate step of solving for the score bidding function, which could involve substantial calculations.
} 
If $s\left(c_{i}\right)$ is an equilibrium, then we must have $\tilde{c}_{i}^{*}=c_{i}$. This gives the following first order condition

$$
\begin{aligned}
& \left.-(N-1)\left[1-F\left(c_{i}\right)\right]^{N-2} f\left(\tilde{c}_{i}\right)\right]\left[p\left(\tilde{c}_{i} \mid s\left(\tilde{c}_{i}\right)\right)-(1-\rho) c_{i} \varsigma\left(q\left(\tilde{c}_{i} \mid s\left(\tilde{c}_{i}\right)\right)\right)\right] \\
& +\left[1-F\left(c_{i}\right)\right]^{N-1}\left[p_{c_{i}}\left(\tilde{c}_{i} \mid s\left(\tilde{c}_{i}\right)\right)+p_{s}\left(\tilde{c}_{i} \mid s\left(\tilde{c}_{i}\right)\right) s^{\prime}\left(\tilde{c}_{i}\right)\right. \\
& \left.\quad-(1-\rho) c_{i} \varsigma^{\prime}\left(q\left(\tilde{c}_{i} \mid s\left(\tilde{c}_{i}\right)\right)\right)\left(q_{c_{i}}\left(\tilde{c}_{i} \mid s\left(\tilde{c}_{i}\right)\right)+q_{s}\left(\tilde{c}_{i} \mid s\left(\tilde{c}_{i}\right)\right) s^{\prime}\left(\tilde{c}_{i}\right)\right)\right] \\
& -\rho c_{i} \varsigma^{\prime}\left(q\left(\tilde{c}_{i} \mid s\left(\tilde{c}_{i}\right)\right)\right)\left(q_{c_{i}}\left(\tilde{c}_{i} \mid s\left(\tilde{c}_{i}\right)\right)+q_{s}\left(\tilde{c}_{i} \mid s\left(\tilde{c}_{i}\right)\right) s^{\prime}\left(\tilde{c}_{i}\right)\right) \\
& =0, \text { when } \tilde{c}_{i}=c_{i} .
\end{aligned}
$$

Under the proper conditions, this equation would pin down a solution of $s\left(c_{i}\right)$ with applicable boundary condition. If $s\left(c_{i}\right)$ is decreasing in type, then the equilibrium is identified as $(q(\cdot), p(\cdot))=\left(q\left(c_{i} \mid s\left(c_{i}\right)\right), p\left(c_{i} \mid s\left(c_{i}\right)\right)\right)$. The procedure proposed in this paper has the merit of saving the middle step of solving for the equilibrium score by first directly solving for the quality bid directly.

\section{Equilibrium when quality bids are placed before price bids}

In this section, we focus on the case of all-pay quality bids, i.e. $\rho=1$. Suppose now that the suppliers must first decide simultaneously which qualities to provide. The quality bids will then be observed by both suppliers before they simultaneously make the second stage price bids. We solve the game by backward induction.

We start from the second stage. Suppose the first round quality bids are $q_{i}, i=1,2$. Clearly, the second stage is a simultaneous move price setting subgame between the two suppliers. We use $q^{(1)}$ and $q^{(2)}$ to denote the higher and lower quality bids from the first stage. The supplier with higher quality $q^{(1)}$ would bid at a level $p$ such that $q^{(1)}-p=q^{(2)}$, and the supplier with lower quality $q^{(2)}$ would bid at a zero price. ${ }^{14}$ The supplier with higher quality wins the procurement and earns a second stage surplus of $p=q^{(1)}-q^{(2)}$.

We now derive the first stage quality bids. Assume a decreasing symmetric bidding strategy $q(\cdot)$. We apply the truthful direct mechanism approach. Assuming that the other supplier follows strategy $q(\cdot)$, the expected payoff of supplier $i$ of type $c_{i}$ is as follows if he reports $c_{i}^{\prime}$ :

$$
\tilde{\pi}_{i}\left(c_{i}^{\prime}, c_{i}\right)=\int_{c_{i}^{\prime}}^{\bar{c}}\left(q\left(c_{i}^{\prime}\right)-q(\tau)\right) f(\tau) d \tau-c_{i} \varsigma\left(q\left(c_{i}^{\prime}\right)\right)
$$

The incentive compatibility condition requires $\left.\frac{\partial \tilde{\pi}_{i}\left(c_{i}^{\prime}, c_{i}\right)}{\partial c_{i}^{\prime}}\right|_{c_{i}^{\prime}=c_{i}}=0$, which yields

$$
\left.q^{\prime}\left(c_{i}^{\prime}\right)\left[\left(1-F\left(c_{i}^{\prime}\right)\right)-c_{i} \varsigma^{\prime}\left(q\left(c_{i}^{\prime}\right)\right)\right]\right|_{c_{i}^{\prime}=c_{i}}=q^{\prime}\left(c_{i}\right)\left[\left(1-F\left(c_{i}\right)\right)-c_{i} \varsigma^{\prime}\left(q\left(c_{i}\right)\right)\right]=0 .
$$

\footnotetext{
${ }^{14}$ We assume that when the procurer is indifferent between the two suppliers' scores, he allocates the contract to the supplier with higher quality. In the event of identical quality, any tie breaking rule works.
} 
This reduces to

$$
\varsigma^{\prime}\left(q\left(c_{i}\right)\right)=\frac{1-F\left(c_{i}\right)}{c_{i}}
$$

which coincides with (8) for $\rho=1$. Therefore, as was established in Section $3, q\left(c_{i}\right)$ is decreasing in type. We thus have that the observability of quality bids when suppliers make their price bids does not change their quality choices.

Moreover, the total surplus, the procurer's expected payoff and each supplier's expected payoff are not affected. It is clear that the total surplus is not affected as this is completely determined by the suppliers' quality choices, since the total surplus is simply the difference between the higher quality and total costs incurred.

The first stage IC condition, together with the envelope theorem, leads to

$$
\frac{d \tilde{\pi}_{i}\left(c_{i}, c_{i}\right)}{d c_{i}}=-\varsigma\left(q\left(c_{i}\right)\right)
$$

Note we still have $\tilde{\pi}_{i}(\bar{c}, \bar{c})=0$ as the least efficient type never wins and always incurs zero cost. We thus have

$$
\pi_{i}\left(c_{i}, c_{i}\right)=\int_{c_{i}}^{\bar{c}} \varsigma(q(t)) d t
$$

which coincides with (5), which gives the suppliers' expected payoff conditional on type $c_{i}$ for the case of simultaneous quality and price bids. Since total surplus and the supplier's expected payoff conditional on type $c_{i}$ are the same across the two scenarios, the procurer's expected payoff must also be the same. We summarize these results in the following theorem.

Theorem 2 Quality bids, supplier expected payoff conditional on type $c_{i}$ and procurer expected payoff (and thus expected total surplus) do not depend on whether quality bids are placed simultaneously with or before the price bids.

\section{$5 \quad$ All-pay versus winner-pay quality bids}

We now turn to the comparison between a procurement auction with all-pay quality bids and two benchmark environments of winner-pay quality bids. In the first benchmark scenario of winner-pay quality bids, we assume a hypothetical environment, in which the losing suppliers do not need to incur costs to place their quality bids. In the second benchmark scenario of winner-pay quality bids, we have both suppliers incur their costs of placing their quality bids but the losing supplier's cost of placing his quality bid is reimbursed by the procurer. 


\subsection{Comparison to the first benchmark: loser incurs no quality cost $(\rho=0)$}

We first compare a procurement auction with all-pay quality bids with the first benchmark of winner-pay quality bids, in which hypothetically the losing suppliers do not need to incur costs to place their quality bids. We will establish that all-pay quality bids lead to lower quality provision in general, and for the class of cost functions $\varsigma(q)=q^{\gamma}, \gamma>1$, lower total surplus and suppliers' payoffs. Moreover, we will identify conditions under which the procurer derives higher and lower payoffs from all-pay quality bids than winner-pay quality bids. In particular, we will show that when $\varsigma(q)=q^{\gamma}$, as $\gamma$ converges to 1 from above, i.e. when the cost function $\varsigma(q)$ gets close to being linear, winner-pay bids $(\rho=0)$ cannot be procurer payoff maximizing if the proportion $\rho$ can be arbitrarily chosen in $[0,1]$.

First, by (9), the equilibrium quality bid decreases in $\rho$, which means that all-pay quality bids lead to lower quality provision.

Proposition 1 All-pay quality bids lead to lower quality provision than winner-pay quality bids.

From (4), we have

$$
\frac{d \pi_{i}\left(c_{i}, c_{i}\right)}{d c_{i}}=-\left\{\left[1-F\left(c_{i}\right)\right](1-\rho)+\rho\right\} \varsigma\left(q\left(c_{i}, \rho\right)\right),
$$

where from (9)

$$
q\left(c_{i} ; \rho\right)=\left(\varsigma^{\prime}\right)^{-1}\left(\frac{1-F\left(c_{i}\right)}{c_{i}\left\{(1-\rho)\left[1-F\left(c_{i}\right)\right]+\rho\right\}}\right) .
$$

Therefore, we have

$$
\frac{d \pi_{i}\left(c_{i}, c_{i}\right)}{d c_{i}}=-\left[1-F\left(c_{i}\right)\right]\left\{\frac{\left[1-F\left(c_{i}\right)\right](1-\rho)+\rho}{1-F\left(c_{i}\right)}\right\}\left(\varsigma \circ\left(\varsigma^{\prime}\right)^{-1}\right)\left(\frac{1-F\left(c_{i}\right)}{c_{i}\left\{(1-\rho)\left[1-F\left(c_{i}\right)\right]+\rho\right\}}\right) .
$$

Since $\pi_{i}(\bar{c}, \bar{c})=0$ in both scenarios, if we can show that $\left|\frac{d \pi_{i}\left(c_{i}, c_{i}\right)}{d c_{i}}\right|$ decreases with $\rho$, then all-pay quality bids lead to lower supplier payoffs than winner-pay quality bids. This is the case when $\varphi=\varsigma \circ\left[\left(\varsigma^{\prime}\right)^{-1}\right]$ is convex as will be verified in the proof of the following proposition. Clearly, $\varsigma(q)=q^{\gamma}, \gamma>1$ satisfies this condition.

Proposition 2 If $\varphi=\varsigma \circ\left(\varsigma^{\prime}\right)^{-1}$ is convex, then all-pay quality bids lead to lower supplier payoffs than winner-pay quality bids.

We next turn to the total surplus and the procurer's payoff. Total surplus is

$$
\begin{aligned}
T S(\rho)= & \int_{\underline{c}}^{\bar{c}} q(c ; \rho) d\left[1-(1-F(c))^{2}\right] \\
& -2 \cdot E_{c_{i}}\left\{(1-\rho) c_{i} \varsigma\left(q\left(c_{i} ; \rho\right)\right)\left[1-F\left(c_{i}\right)\right]\right\}-2 \cdot E_{c_{i}} \rho c_{i} \varsigma\left(q\left(c_{i} ; \rho\right)\right) .
\end{aligned}
$$

The procurer's payoff equals the difference between total surplus and the suppliers' payoffs. A supplier's 
expected payoff is

$$
\begin{aligned}
\pi_{S}(\rho) & =\int_{\underline{c}}^{\bar{c}} \pi_{i}\left(c_{i}, c_{i}\right) d F\left(c_{i}\right) \\
& =\int_{\underline{c}}^{\bar{c}} \int_{c_{i}}^{\bar{c}}\{[1-F(t)](1-\rho)+\rho\} \varsigma(q(t ; \rho)) d t d F\left(c_{i}\right) .
\end{aligned}
$$

Therefore, the procurer's payoff equals ${ }^{15}$

$$
\pi_{P}(\rho)=T S(\rho)-2 \pi_{S}(\rho)
$$

In the following analysis, we consider $\varsigma(q)=q^{\gamma}, \gamma>1$. With this specification, we have the following results.

Lemma 2 Assume $\varsigma(q)=q^{\gamma}, \gamma>1$. We have

$$
\begin{aligned}
T S(\rho) & =2\left(1-\frac{1}{\gamma}\right) E_{c}\left\{\left[\frac{1}{\gamma c\left[(1-\rho)+\frac{\rho}{1-F(c)}\right]}\right]^{\frac{1}{\gamma-1}}(1-F(c))\right\} ; \\
\pi_{S}(\rho) & =E_{c}\left\{\frac{1}{\gamma c}\left[\frac{1}{\gamma c\left[(1-\rho)+\frac{\rho}{1-F(c)}\right]}\right]^{\frac{1}{\gamma-1}} \frac{F(c)}{f(c)}[1-F(c)]\right\} ; \\
\pi_{P}(\rho) & =2 E_{c}\left\{\left[\frac{1}{\gamma c\left[(1-\rho)+\frac{\rho}{1-F(c)}\right]}\right]^{\frac{1}{\gamma-1}}(1-F(c))\left[\left(1-\frac{1}{\gamma}\right)-\frac{1}{\gamma c} \frac{F(c)}{f(c)}\right]\right\} .
\end{aligned}
$$

Lemma 2 immediately leads to the following results. ${ }^{16}$

Proposition 3 If $\varsigma=q^{\gamma}, \gamma>1$, all-pay quality bids lead to lower total surplus and suppliers' payoffs than winner-pay bids; and they lead to lower procurer payoff when $\left(1-\frac{1}{\gamma}\right)-\frac{1}{\gamma c} \frac{F(c)}{f(c)} \geq 0, \forall c \in[\underline{c}, \bar{c}]$.

For example, we consider the class $F(c)=(c-1)^{\eta}$ on $[1,2], \eta>0$. For this class of type distributions, we have

${ }^{15}$ This can be verified as below:

$$
\begin{aligned}
& \pi_{P}(\rho)=\int_{\underline{c}}^{\bar{c}}(q(c)-p(c)) 2(1-F(c)) d F(c)=2 \int_{\underline{c}}^{\bar{c}}[q(c)(1-F(c))-p(c)(1-F(c))] d F(c) \\
& =2 \int_{\underline{c}}^{\bar{c}}\left\{q(c)(1-F(c))-\int_{c}^{\bar{c}}\{[(1-F(t))(1-\rho)+\rho] \varsigma(q(t))\} d t-\varsigma(q(c)) c[\rho+(1-\rho)(1-F(c))]\right\} d F(c) \\
& =\underbrace{2 \int_{\underline{c}}^{\bar{c}}\{q(c)(1-F(c))-\varsigma(q(c)) c[\rho+(1-\rho)(1-F(c))]\} d F(c)}_{T S(\rho)} \\
& -2 \underbrace{-2 \int_{\underline{c}}^{\bar{c}} \int_{c}^{\bar{c}}\{[(1-F(t))(1-\rho)+\rho] \varsigma(q(t))\} d t d F(c)}_{\pi_{S}(\rho)} .
\end{aligned}
$$

${ }^{16}$ The result on suppliers' payoffs in Proposition 3 is consistent with that of Proposition 2. 


$$
\left(1-\frac{1}{\gamma}\right)-\frac{1}{\gamma c} \frac{F(c)}{f(c)}=\left(1-\frac{1}{\gamma}\right)-\frac{1}{\eta \gamma} \frac{c-1}{c} .
$$

Since $\frac{c-1}{c}<1$, the right hand side of the above equation is always positive when

$$
\left(1-\frac{1}{\gamma}\right)-\frac{1}{\eta \gamma}>0 \text {, i.e. } 1+\frac{1}{\eta}<\gamma .
$$

We thus have the following corollary. ${ }^{17}$

Corollary 1 If $\varsigma(q)=q^{\gamma}, \gamma>1$, and $F(c)=(c-1)^{\eta}$ on $[1,2]$ with $1+\frac{1}{\eta}<\gamma$, then all-pay quality bids lead to lower procurer payoffs than winner-pay quality bids.

The next proposition reveals a sufficient condition on the cost of quality function that guarantees that winner-pay bids do not maximize the procurer's payoff.

Proposition 4 If $\varsigma=q^{\gamma}, \gamma>1$, then $\pi_{P}^{\prime}(0)$ approaches positive infinity when $\gamma$ approaches 1 from above.

Proposition 4 reveals that if the proportion $\rho$ can be chosen from $[0,1]$ without restriction, then in general winner-pay quality bids do not maximize procurer's payoff if $\gamma$ is sufficiently close to 1 .

We next answer the question whether it is possible that all-pay quality bids lead to a higher procurer payoff than winner-pay quality bids, which would justify the adoption of score procurements with all-pay quality bids. The following analysis illustrates that this is in general possible.

\section{All-pay quality bids can either benefit or hurt the procurer}

Although Proposition 4 reveals a weak condition under which winner-pay quality bids do not maximize the procurer's payoff when the proportion $\rho$ can be arbitrarily chosen from $[0,1]$, it turns out that it is difficult to identify general sufficient conditions under which all-pay quality bids benefit the procurer compared to winner-pay quality bids. We next provide examples to illustrate that all-pay quality bids may indeed benefit the procurer.

Let $\varsigma(q)=q^{\gamma}, \gamma>1, f(c)=4-2 c$ and $F(c)=2\left(2 c-\frac{c^{2}}{2}-\frac{3}{2}\right)$ on $[1,2]$. We have $\frac{F(c)}{c f(c)}=\frac{2 c-\frac{c^{2}}{2}-\frac{3}{2}}{c(2-c)}$ and

${ }^{17}$ Note that $\gamma c\left[(1-\rho)+\frac{\rho}{1-F(c)}\right]$ increases with $\rho$ for any $c$. 
$1-F(c)=(2-c)^{2}$. Note that ${ }^{18}$

$$
\begin{aligned}
& \pi_{P}(\rho=0)=2 E_{c}\left\{\left[\frac{1}{\gamma c}\right]^{\frac{1}{\gamma-1}}(1-F(c))\left[\left(1-\frac{1}{\gamma}\right)-\frac{1}{\gamma} \frac{F(c)}{c f(c)}\right]\right\}, \text { and } \\
& \pi_{P}(\rho=1)=2 E_{c}\left\{\left[\frac{1-F(c)}{\gamma c}\right]^{\frac{1}{\gamma-1}}(1-F(c))\left[\left(1-\frac{1}{\gamma}\right)-\frac{1}{\gamma c} \frac{F(c)}{f(c)}\right]\right\} .
\end{aligned}
$$

Numerical simulation reveals that all-pay quality bids lead to higher procurer payoff than winner-pay quality bids, i.e. $\pi_{p}(\rho=1)>\pi_{p}(\rho=0)$, if and only if $\gamma \in(1,1.18613)$, i.e. when the effort cost function is not far from a linear function. In particular, when $\gamma=1.1$, we have $\pi_{P}(\rho=0)=8.28 \times 10^{-4}$ and $\pi_{P}(\rho=1)=1.52 \times 10^{-3}$.

All-pay quality bids lead to lower quality bids than winner-pay quality bids, which allows suppliers to lower their price bids. It is thus possible that the drop in price bids dominates the drop in quality bids, which makes it possible that all-pay quality bids lead to higher procurer payoff.

\subsection{Comparison to the second benchmark: loser's sunk quality cost reimbursed by the procurer}

We next compare a procurement auction with all-pay quality bids with the second benchmark of winner-pay quality bids, in which both suppliers incur their costs of placing their quality bids but the losing supplier's cost of placing his quality bid is reimbursed by the procurer.

Note that suppliers' payoff functions are identical in the first and second benchmark environments. Therefore, we must have the following result.

Lemma 3 The equilibrium bidding strategy is the same across the two benchmark environments.

Based on Lemma 3 and the results of Section 5.1, we immediately have the following result.

$$
\begin{aligned}
& { }^{{ }^{18} \text { Let } a=\frac{1}{1-\gamma} \text {. One can verify that }} \begin{aligned}
\pi_{p}(\rho=0)= & 2 \gamma^{a} \int_{1}^{2}\left(1-\frac{1}{\gamma}\right) c^{a}(2-c)^{2}-\frac{1}{\gamma} c^{a}(2-c)\left(2-\frac{c}{2}-\frac{3}{2 c}\right) d c \\
= & -\left(1-\frac{1}{a}\right)^{a-1}\left\{\frac{2^{a+5}-4}{a(a+1)(a+2)(a+3)}-\frac{4}{a(a+1)(a+2)}-\frac{2^{a+1} 3-1}{a(a+1)}\right. \\
& \left.+\frac{2^{a+4}-4}{(a+1)(a+2)}-\frac{2^{a+3}-1}{(a+2)(a+3)}+\frac{3}{a}-\frac{4}{a+1}+\frac{1}{a+2}\right\} ; \text { and } \\
\pi_{p}(\rho=1)= & 2 \gamma^{a} \int_{1}^{2}\left(1-\frac{1}{\gamma}\right) c^{a}(2-c)^{2-2 a}-\frac{1}{\gamma} c^{a}(2-c)^{1-2 a}\left(2-\frac{c}{2}-\frac{3}{2 c}\right) d c \\
= & -2\left(1-\frac{1}{a}\right)^{a-1}\left\{\frac{2^{3-a}}{a} \beta_{\frac{1}{2}}(3-2 a, 1+a)+2^{3-a} \beta_{\frac{1}{2}}(2-2 a, 1+a)\right. \\
& \left.-2^{2-a} \beta_{\frac{1}{2}}(2-2 a, 2+a)-\frac{3}{2^{a}} \beta_{\frac{1}{2}}(2-2 a, a)\right\},
\end{aligned}
\end{aligned}
$$

where $\beta_{z}(d, e)=\int_{0}^{z} x^{d-1}(1-x)^{e-1} d x$ is the beta function. 
Proposition 5 Compared to the second benchmark with winner-pay quality bids, (i) all-pay quality bids lead to lower quality provision; (ii) if $\varphi=\varsigma \circ\left(\varsigma^{\prime}\right)^{-1}$ is convex (e.g. when $\varsigma(q)=q^{\gamma}, \gamma>1$ ), then all-pay quality bids lead to lower supplier payoffs.

Note that equilibrium bidding strategies are the same across the two benchmark environments as shown by Lemma 3, and in the second benchmark, the loser also incurs a quality provision cost that the procurer covers. We thus have the following results.

Corollary 2 The suppliers' payoffs must be same across the two benchmark environments, and the total surplus and procurer's payoff must be lower in the second benchmark environment.

This means that a procurement auction with all-pay quality bids is more likely to render a higher procurer payoff when the second benchmark of winner-pay quality bids is adopted instead of the first benchmark. In particular, in the examples provided in the end of Section 5.1, all-pay quality bids generate higher procurer payoffs than winner-pay quality bids under the second benchmark, whenever they do under the first benchmark.

It remains to further investigate whether the second winner-pay benchmark can generate a higher procurer payoff than all-pay quality bids, and how the total surplus compares. We investigate these issues under the assumption that $\varsigma(q)=q^{\gamma}, \gamma>1$. Note that under this specification, we have that $q(c ; \rho=0)=(\gamma c)^{-\frac{1}{\gamma-1}}$ by $(9)$ and thus $\varsigma(q(c ; \rho=0))=(\gamma c)^{-\frac{\gamma}{\gamma-1}}$.

We first look at total surplus. In a procurement auction with all-pay quality bids, i.e. $\rho=1$, by Lemma 2 , the total surplus is

$$
\begin{aligned}
T S(1) & =2\left(1-\frac{1}{\gamma}\right) E_{c}\left\{\left[\frac{1-F(c)}{\gamma c}\right]^{\frac{1}{\gamma-1}}(1-F(c))\right\} \\
& =2(\gamma-1) \gamma^{-\frac{\gamma}{\gamma-1}} E_{c}\left[c^{-\frac{1}{\gamma-1}}(1-F(c))^{\frac{\gamma}{\gamma-1}}\right] .
\end{aligned}
$$

Let $c^{(1)}$ denote the higher cost parameter of the suppliers. In the second benchmark of winner-pay quality bids, in which both suppliers incur their costs of placing their quality bids but the losing supplier's cost of placing his quality bid is reimbursed by the procurer, the total surplus is

$$
\begin{aligned}
T S^{R}(1) & =T S(0)-E_{c^{(1)}}\left[c^{(1)} \varsigma\left(q\left(c^{(1)} ; \rho=0\right)\right)\right] \\
& =2\left(1-\frac{1}{\gamma}\right) E_{c}\left\{\left[\frac{1}{\gamma c}\right] \frac{1}{\gamma-1}(1-F(c))\right\}-2 E_{c}[c \varsigma(q(c ; \rho=0)) F(c)] \\
& =2\left(1-\frac{1}{\gamma}\right) E_{c}\left\{\left[\frac{1}{\gamma c}\right] \frac{1}{\gamma-1}(1-F(c))\right\}-2 E_{c}\left[\gamma^{-\frac{\gamma}{\gamma-1}} c^{-\frac{1}{\gamma-1}} F(c)\right] \\
& =2(\gamma-1) \gamma^{-\frac{\gamma}{\gamma-1}} E_{c}\left[c^{-\frac{1}{\gamma-1}}(1-F(c))\right]-2 E_{c}\left[\gamma^{-\frac{\gamma}{\gamma-1}} c^{-\frac{1}{\gamma-1}} F(c)\right] .
\end{aligned}
$$


We thus have

$$
\frac{T S^{R}(1)-T S(1)}{2 \gamma^{-\frac{\gamma}{\gamma-1}}(\gamma-1)}=E_{c}\left\{c^{-\frac{1}{\gamma-1}}\left[(1-F(c))-(1-F(c))^{\frac{\gamma}{\gamma-1}}-\frac{1}{\gamma-1} F(c)\right]\right\} .
$$

Let $x=F(c) \in[0,1]$. Note $\left[(1-x)-(1-x)^{\frac{\gamma}{\gamma-1}}-\frac{1}{\gamma-1} x\right]^{\prime}=\frac{\gamma}{\gamma-1}\left[(1-x)^{\frac{1}{\gamma-1}}-1\right] \leq 0$ and $(1-x)-(1-$ $x)^{\frac{\gamma}{\gamma-1}}-\frac{1}{\gamma-1} x=0$ when $x=0$. Therefore, $(1-F(c))-(1-F(c))^{\frac{\gamma}{\gamma-1}}-\frac{1}{\gamma-1} F(c) \leq 0, \forall c$. We thus have

$$
T S^{R}(1)-T S(1) \leq 0
$$

Recall that Proposition 5 says that all-pay quality bids lead to lower suppliers' payoffs than the second benchmark scenario if $\varsigma(q)=q^{\gamma}, \gamma>1$. This implies that the procurer must enjoy a higher payoff with all-pay quality bids than with winner-pay bids under the second benchmark.

We summarize the above results as follows.

Proposition 6 Suppose $\varsigma=q^{\gamma}, \gamma>1$. Relative to the second benchmark environment where the losing supplier's cost of placing his quality bid is reimbursed by the procurer, all-pay quality bids generate higher total surplus and higher procurer payoffs.

\section{Concluding remarks}

In this paper, we study the impact of an all-pay quality component in score procurements. Our focus is on the equilibrium quality, supplier payoff, procurer payoff and total surplus. We find that compared to benchmark environments with winner-pay quality bids, all-pay quality bids tend to lower the quality provision. However, all-pay quality bids may improve the procurer's payoff. This finding thus provides an additional justification for a procurer's requirement that suppliers actually incur the cost of developing the product as part of their bid. Doing so not only guarantees that the procurer acquires exactly the quality that the winning supplier bids, but also may increase the procurer's payoff.

Surprisingly, our study shows that when all-pay quality bids are placed and disclosed before price bids, the same quality bids, suppliers' payoffs and procurer's payoff are generated as in the case of simultaneous quality and price bids. One implication of this finding is that when price bids are simultaneously placed after simultaneous all-pay quality bids, a policy of revelation of the quality bid information before suppliers make their price bids does not alter equilibrium quality and expected payoffs of the procurer and suppliers. ${ }^{19}$

One methodological innovation in this paper lies in that we come up with a two-step procedure, which allows us to identify the quality and price bids directly if there exists a symmetric pure strategy equilibrium that renders scores decreasing in types. This procedure does not rely on solving for the equilibrium score function as a middle step. Our procedure also reveals if such an equilibrium does not exist.

\footnotetext{
${ }^{19}$ Despite identical expected transfers from the procurer to the suppliers under the two informational regimes (due to payoff equivalence), the distribution of the transfer conditional on the type profile differs.
} 


\section{Appendix A}

\section{Proof of Lemma 1}

Note that from (6), we have

$$
\left(1-F\left(c_{i}\right)\right) p\left(c_{i}\right)=\int_{c_{i}}^{\bar{c}}\{[1-F(t)](1-\rho) \varsigma(q(t))+\rho \varsigma(q(t))\} d t+\rho c_{i} \varsigma\left(q\left(c_{i}\right)\right)+\left(1-F\left(c_{i}\right)\right)(1-\rho) c_{i} \varsigma\left(q\left(c_{i}\right)\right) .
$$

Note that from (8), we have $\frac{c_{i}\left[\rho+(1-\rho)\left(1-F\left(c_{i}\right)\right)\right]}{1-F\left(c_{i}\right)} \varsigma^{\prime}\left(q\left(c_{i}\right)\right)=1$. We thus have

$$
\begin{aligned}
& {\left[\left(1-F\left(c_{i}\right)\right) p\left(c_{i}\right)\right]^{\prime}=\rho c_{i} \varsigma^{\prime}\left(q\left(c_{i}\right)\right) q^{\prime}\left(c_{i}\right)+c_{i} \frac{d\left\{\left[1-F\left(c_{i}\right)\right](1-\rho) \varsigma\left(q\left(c_{i}\right)\right)\right\}}{d c_{i}} } \\
\Leftrightarrow & \left(1-F\left(c_{i}\right)\right) p^{\prime}\left(c_{i}\right)-f\left(c_{i}\right) p\left(c_{i}\right)=\rho c_{i} \varsigma^{\prime}\left(q\left(c_{i}\right)\right) q^{\prime}\left(c_{i}\right)+c_{i} \frac{d\left\{\left[1-F\left(c_{i}\right)\right](1-\rho) \varsigma\left(q\left(c_{i}\right)\right)\right\}}{d c_{i}} \\
\Leftrightarrow & p^{\prime}\left(c_{i}\right)-\frac{f\left(c_{i}\right)}{1-F\left(c_{i}\right)} p\left(c_{i}\right)=q^{\prime}\left(c_{i}\right) \frac{c_{i}\left[\rho+(1-\rho)\left(1-F\left(c_{i}\right)\right)\right]}{1-F\left(c_{i}\right)} \varsigma^{\prime}\left(q\left(c_{i}\right)\right)-\frac{f\left(c_{i}\right)}{1-F\left(c_{i}\right)} c_{i}(1-\rho) \varsigma\left(q\left(c_{i}\right)\right) \\
\Leftrightarrow & p^{\prime}\left(c_{i}\right)=q^{\prime}\left(c_{i}\right)-\frac{f\left(c_{i}\right)}{1-F\left(c_{i}\right)} c_{i}(1-\rho) \varsigma\left(q\left(c_{i}\right)\right)+\frac{f\left(c_{i}\right)}{1-F\left(c_{i}\right)} p\left(c_{i}\right) \\
\Leftrightarrow & s^{\prime}\left(c_{i}\right)=q^{\prime}\left(c_{i}\right)-p^{\prime}\left(c_{i}\right)=\frac{f\left(c_{i}\right)}{1-F\left(c_{i}\right)}\left\{c_{i}(1-\rho) \varsigma\left(q\left(c_{i}\right)\right)-p\left(c_{i}\right)\right\} .
\end{aligned}
$$

Note that from (6), we have $c_{i}(1-\rho) \varsigma\left(q\left(c_{i}\right)\right)-p\left(c_{i}\right)<0$. We thus have $s^{\prime}\left(c_{i}\right)<0$.

\section{Proof of Proposition 2}

Recall

$$
\frac{d \pi_{i}\left(c_{i}, c_{i}\right)}{d c_{i}}=-\left[1-F\left(c_{i}\right)\right]\left\{\frac{\left[1-F\left(c_{i}\right)\right](1-\rho)+\rho}{1-F\left(c_{i}\right)}\right\}\left(\varsigma \circ\left(\varsigma^{\prime}\right)^{-1}\right)\left(\frac{1-F\left(c_{i}\right)}{c_{i}\left\{(1-\rho)\left[1-F\left(c_{i}\right)\right]+\rho\right\}}\right) .
$$

Since $\pi_{i}(\bar{c}, \bar{c})=0$ in both scenarios, if we can show that $\left|\frac{d \pi_{i}\left(c_{i}, c_{i}\right)}{d c_{i}}\right|$ decreases with $\rho$, then we have that all-pay quality bids lead to lower supplier payoff than winner-pay quality bids.

Let $x(\rho)=\frac{1-F\left(c_{i}\right)}{c_{i}\left\{(1-\rho)\left[1-F\left(c_{i}\right)\right]+\rho\right\}}$. We have $x(\rho)>0, x^{\prime}(\rho)<0$. Thus the monotonicity of $\left|\frac{d \pi_{i}\left(c_{i}, c_{i}\right)}{d c_{i}}\right|$ with respect to $\rho$ is equivalent to the monotonicity of $\frac{1}{x} \varphi(x), x \geq 0$. Note $\varphi(0)=0$ and $\lim _{x \rightarrow 0} \frac{1}{x} \varphi(x)=\varphi^{\prime}(0) \geq 0$, since $\varphi$ increases. In addition, $\left[\frac{1}{x} \varphi(x)\right]^{\prime}=\frac{1}{x^{2}}\left[x \varphi^{\prime}(x)-\varphi(x)\right] \cdot x \varphi^{\prime}(x)-\varphi(x)$ increases with $x$ since its derivative is $x \varphi^{\prime \prime}(x) \geq 0$ and reaches zero when $x=0$. We thus have $\left[\frac{1}{x} \varphi(x)\right]^{\prime} \geq 0$, i.e. $\left|\frac{d \pi_{i}\left(c_{i}, c_{i}\right)}{d c_{i}}\right|$ decreases with $\rho$.

\section{Proof of Lemma 2}

By (9), we have

$$
q\left(c_{i} ; \rho\right)=\left[\frac{1}{\gamma c_{i}\left[(1-\rho)+\frac{\rho}{1-F\left(c_{i}\right)}\right]}\right]^{\frac{1}{\gamma-1}} .
$$


The expected total surplus is thus

$$
\begin{aligned}
T S(\rho)= & \int_{\underline{c}}^{\bar{c}} q(\tau ; \rho) d\left[1-(1-F(\tau))^{2}\right]-2 \cdot E_{c_{i}}\left\{(1-\rho) c_{i} \varsigma\left(q\left(c_{i} ; \rho\right)\right)\left[1-F\left(c_{i}\right)\right]\right\}-2 \cdot E_{c_{i}}\left[\rho c_{i} \varsigma\left(q\left(c_{i} ; \rho\right)\right)\right] \\
= & 2 E_{\tau}\left\{\left[\frac{1}{\gamma \tau\left[(1-\rho)+\frac{\rho}{1-F(\tau)}\right]}\right]^{\frac{1}{\gamma-1}}(1-F(\tau))\right\}-2 \cdot E_{\tau}\left\{(1-\rho) \tau\left[\frac{1}{\gamma \tau\left[(1-\rho)+\frac{\rho}{1-F(\tau)}\right]}\right]^{\frac{\gamma}{\gamma-1}}[1-F(\tau)]\right\} \\
& -2 \cdot E_{\tau}\left\{\rho \tau\left[\frac{1}{\gamma \tau\left[(1-\rho)+\frac{\rho}{1-F(\tau)}\right]}\right]^{\frac{\gamma}{\gamma-1}}\right\} \\
= & 2 E_{\tau}\left\{\left[\frac{1}{\gamma \tau\left[(1-\rho)+\frac{\rho}{1-F(\tau)}\right]}\right]^{\frac{1}{\gamma-1}}(1-F(\tau))\right\} \\
& -2 \cdot E_{\tau}\left\{\left[\frac{1}{\gamma \tau\left[(1-\rho)+\frac{\rho}{1-F(\tau)}\right]}\right]^{\frac{\gamma}{\gamma-1}} \tau\left[(1-\rho)-\frac{\rho}{1-F(\tau)}\right][1-F(\tau)]\right\} \\
= & 2 E_{\tau}\left\{\left[\frac{1}{\gamma \tau\left[(1-\rho)+\frac{\rho}{1-F(\tau)}\right]}\right]^{\frac{1}{\gamma-1}}(1-F(\tau))\right\}-2 \cdot E_{\tau}\left\{\left[\frac{1}{\gamma \tau\left[(1-\rho)+\frac{\rho}{1-F(\tau)}\right]}\right]^{\left.\frac{1}{\gamma-1}[1-F(\tau)] \frac{1}{\gamma}\right\}}\right. \\
= & 2\left(1-\frac{1}{\gamma}\right) E_{\tau}\left\{\left[\frac{1}{\gamma \tau\left[(1-\rho)+\frac{\rho}{1-F(\tau)}\right]}\right]^{\frac{1}{\gamma-1}}(1-F(\tau))\right\} .
\end{aligned}
$$

Suppliers' expected surplus is

$$
\begin{aligned}
& 2 \pi_{S}(\rho) \\
= & 2 \int_{\underline{c}}^{\bar{c}} \int_{c}^{\bar{c}}\{[1-F(\tau)](1-\rho)+\rho\} \varsigma(q(\tau ; \rho)) d \tau d F(c) \\
= & 2 \int_{\underline{c}}^{\bar{c}} \int_{0}^{\tau}\{[1-F(\tau)](1-\rho)+\rho\} \varsigma(q(\tau)) d F(c) d \tau \\
= & 2 \int_{\underline{c}}^{\bar{c}}\{[1-F(\tau)](1-\rho)+\rho\} \varsigma(q(\tau)) F(\tau) d \tau \\
= & 2 E_{\tau}\left\{\left[(1-\rho)+\frac{\rho}{1-F(\tau)}\right]\left[\frac{\rho}{\gamma \tau\left[(1-\rho)+\frac{\rho}{1-F(\tau)}\right]}\right] \frac{\gamma}{\gamma-1} \frac{F(\tau)}{f(\tau)}[1-F(\tau)]\right\} \\
= & 2 E_{\tau}\left\{\left[(1-\rho)+\frac{\rho}{1-F(\tau)}\right]\left[\frac{1}{\gamma \tau\left[(1-\rho)+\frac{\rho}{1-F(\tau)}\right]}\right]\right. \\
& \left.\cdot\left[\frac{1}{\gamma \tau\left[(1-\rho)+\frac{\rho}{1-F(\tau)}\right]}\right] \frac{1}{\gamma-1} \frac{F(\tau)}{f(\tau)}[1-F(\tau)]\right\} \\
= & 2 E_{\tau}\left\{\left[\frac{1}{\gamma \tau}\right]\left[\frac{1}{\gamma \tau\left[(1-\rho)+\frac{\rho}{1-F(\tau)}\right]}\right] \frac{1}{\gamma-1} \frac{F(\tau)}{f(\tau)}[1-F(\tau)]\right\} .
\end{aligned}
$$


Therefore, we have the following procurer's expected payoff

$$
\begin{aligned}
\pi_{P}(\rho)= & T S(\rho)-2 \pi_{S}(\rho) \\
= & 2\left(1-\frac{1}{\gamma}\right) E_{\tau}\left[\frac{1}{\gamma \tau\left[(1-\rho)+\frac{\rho}{1-F(\tau)}\right]}\right]^{\frac{1}{\gamma-1}}(1-F(\tau)) \\
& -2 E_{\tau}\left[\frac{1}{\gamma \tau}\right]\left[\frac{1}{\gamma \tau\left[(1-\rho)+\frac{\rho}{1-F(\tau)}\right]}\right]^{\frac{1}{\gamma-1}} \frac{F(\tau)}{f(\tau)}[1-F(\tau)] \\
= & 2 E_{\tau}\left[\frac{1}{\gamma \tau\left[(1-\rho)+\frac{\rho}{1-F(\tau)}\right]}\right]^{\frac{1}{\gamma-1}}(1-F(\tau))\left[\left(1-\frac{1}{\gamma}\right)-\frac{1}{\gamma \tau} \frac{F(\tau)}{f(\tau)}\right] .
\end{aligned}
$$

\section{Proof of Proposition 4}

We have

$$
\pi_{P}^{\prime}(\rho)=2 E_{\tau}\left\{\left(-\frac{1}{\gamma-1}\right)\left[\gamma \tau\left[(1-\rho)+\frac{\rho}{1-F(\tau)}\right]^{-\gamma} F(\tau)\left[\left(1-\frac{1}{\gamma}\right)-\frac{1}{\gamma \tau} \frac{F(\tau)}{f(\tau)}\right]\right\}\right.
$$

Thus

$$
\pi_{P}^{\prime}(0)=2 E_{\tau}\left\{\left(-\frac{1}{\gamma-1}\right)[\gamma \tau]^{-\gamma} F(\tau)\left[\left(1-\frac{1}{\gamma}\right)-\frac{1}{\gamma \tau} \frac{F(\tau)}{f(\tau)}\right]\right\}
$$

Therefore

$$
\lim _{\gamma \rightarrow 1^{+}} \pi_{P}^{\prime}(0)(\gamma-1)=2 \int\left[\frac{F(\tau)}{\tau}\right]^{2} d \tau>0
$$

We thus have

$$
\lim _{\gamma \rightarrow 1^{+}} \pi_{P}^{\prime}(0)=+\infty
$$




\section{Appendix B}

In this section, we describe how to generalize our two-step procedure for identifying symmetric pure strategy equilibrium in an environment with $N \geq 2$ suppliers and a general score function $s(q, p)$ with $s_{q}(q, p)>0$, $s_{p}(q, p)<0$.

\section{Implication of the first observation}

We treat equilibrium $\left(q\left(c_{i}\right), p\left(c_{i}\right)\right)$ as an incentive compatible general direct mechanism that generates scores decreasing in types, then the Myerson [11] approach allows us to write down payment rule $p\left(c_{i}\right)$ as a function of the allocation rule $q\left(c_{i}\right)$. The following is the details.

Given $\left(q\left(c_{i}\right), p\left(c_{i}\right)\right)$ that leads to scores that are decreasing in types, consider a type $c_{i}$ supplier $i$ 's problem of making an announcement $\tilde{c}_{i}$ to maximize his expected payoff when the other supplier is truthful:

$$
\max _{\tilde{c}_{i}} \pi_{i}\left(\tilde{c}_{i}, c_{i}\right)=\left[1-F\left(\tilde{c}_{i}\right)\right]^{N-1}\left[p\left(\tilde{c}_{i}\right)-(1-\rho) c_{i} \varsigma\left(q\left(\tilde{c}_{i}\right)\right)\right]-\rho c_{i} \varsigma\left(q\left(\tilde{c}_{i}\right)\right) .
$$

If $(q(\cdot), p(\cdot))$ is a pure strategy equilibrium, we would have $\tilde{c}_{i}^{*}=c_{i}$, i.e. the incentive compatibility (IC) condition:

$$
c_{i}=\arg \max _{\tilde{c}_{i}} \pi_{i}\left(\tilde{c}_{i}, c_{i}\right)=\left[1-F\left(\tilde{c}_{i}\right)\right]^{N-1}\left[p\left(\tilde{c}_{i}\right)-(1-\rho) c_{i} \varsigma\left(q\left(\tilde{c}_{i}\right)\right)\right]-\rho c_{i} \varsigma\left(q\left(\tilde{c}_{i}\right)\right) .
$$

The IC condition (12), together with the envelope theorem, thus leads to

$$
\frac{d \pi_{i}\left(c_{i}, c_{i}\right)}{d c_{i}}=-\left\{\left[1-F\left(c_{i}\right)\right]^{N-1}(1-\rho) \varsigma\left(q\left(c_{i}\right)\right)+\rho \varsigma\left(q\left(c_{i}\right)\right)\right\} .
$$

Note that at equilibrium with scores monotonic in types, we must have $\pi_{i}(\bar{c}, \bar{c})=0$ for both $\rho=0$ and $\rho=1$. For both cases, the least efficient type $\bar{c}$ never wins and never incurs cost to produce. Note we must have $q(\bar{c})=0$ for $\rho=1$.

Using the envelope condition of (13), we have

$$
\pi_{i}\left(c_{i}, c_{i}\right)=\int_{c_{i}}^{\bar{c}}\left\{[1-F(t)]^{N-1}(1-\rho) \varsigma(q(t))+\rho \varsigma(q(t))\right\} d t .
$$

By the definition of $\pi_{i}\left(\tilde{c}_{i}, c_{i}\right)$, we have

$$
\begin{aligned}
\pi_{i}\left(c_{i}, c_{i}\right) & =\left[1-F\left(c_{i}\right)\right]^{N-1}\left[p\left(c_{i}\right)-(1-\rho) c_{i} \varsigma\left(q\left(c_{i}\right)\right)\right]-\rho c_{i} \varsigma\left(q\left(c_{i}\right)\right) \\
& =\int_{c_{i}}^{\bar{c}}\left\{\left[1-F\left(c_{i}\right)\right]^{N-1}(1-\rho) \varsigma\left(q\left(c_{i}\right)\right)+\rho \varsigma\left(q\left(c_{i}\right)\right)\right\} d t
\end{aligned}
$$

which leads to price rule of

$$
p\left(c_{i}\right)=\frac{\int_{c_{i}}^{\bar{c}}\left\{[1-F(t)]^{N-1}(1-\rho) \varsigma(q(t))+\rho \varsigma(q(t))\right\} d t+\rho c_{i} \varsigma\left(q\left(c_{i}\right)\right)}{\left[1-F\left(c_{i}\right)\right]^{N-1}}+(1-\rho) c_{i} \varsigma\left(q\left(c_{i}\right)\right)>0 .
$$




\section{Implication of the second observation}

Suppose $\left(q\left(c_{i}\right), p\left(c_{i}\right)\right)$ is a symmetric pure strategy equilibrium. By the implications of the first observation, we must have $q^{\prime}\left(c_{i}\right)<0$ (monotone allocation rule is implied by incentive compatibility condition) and (15) holds. Define

$$
s\left(c_{i}\right)=s\left(q\left(c_{i}\right), p\left(c_{i}\right)\right)
$$

We now look at the implications of the second observation, which says that equilibrium $\left(q\left(c_{i}\right), p\left(c_{i}\right)\right)$ must solve the following optimization problem:

$$
\begin{aligned}
\left(q\left(c_{i}\right), p\left(c_{i}\right)\right) & =\arg \max _{(q, p)}\left[1-F\left(c_{i}\right)\right]^{N-1}\left[p-(1-\rho) c_{i} \varsigma(q)\right]-\rho c_{i} \varsigma(q), \\
\text { s.t. } & : \quad s(q, p)=s\left(c_{i}\right) .
\end{aligned}
$$

The corresponding Lagrangian is

$$
\max _{(q, p, \lambda)} L(q, p, \lambda)=\left[1-F\left(c_{i}\right)\right]^{N-1}\left[p-(1-\rho) c_{i} \varsigma(q)\right]-\rho c_{i} \varsigma(q)+\lambda\left[s(q, p)-s\left(c_{i}\right)\right] .
$$

The first order conditions are as follows:

$$
\begin{aligned}
& \frac{L(q, p, \lambda)}{\partial q}=-\left[1-F\left(c_{i}\right)\right]^{N-1}(1-\rho) c_{i} \varsigma^{\prime}(q)-\rho c_{i} \varsigma^{\prime}(q)+\lambda s_{q}(q, p)=0, \\
& \frac{L(q, p, \lambda)}{\partial p}=\left[1-F\left(c_{i}\right)\right]^{N-1}+\lambda s_{p}(q, p)=0, \\
& \frac{L(q, p, \lambda)}{\partial \lambda}=s(q, p)-s\left(c_{i}\right)=0 .
\end{aligned}
$$

We thus have

$$
\varsigma^{\prime}\left(q\left(c_{i}\right)\right)=-\frac{s_{q}\left(q\left(c_{i}\right), p\left(c_{i}\right)\right)}{s_{p}\left(q\left(c_{i}\right), p\left(c_{i}\right)\right)} \frac{1}{c_{i}\left\{(1-\rho)+\frac{\rho}{\left[1-F\left(c_{i}\right)\right]^{N-1}}\right\}},
$$

where $p\left(c_{i}\right)$ is given by $(15)$.

Together with boundary condition $q(\bar{c})=0$, we can pin down the candidate quality bid $q\left(c_{i}\right)$. If there is no solution exists, then there is no symmetric pure strategy equilibrium.

Suppose $q\left(c_{i}\right)$ is a solution of (17) with boundary condition $q(\bar{c})=0$, and $p\left(c_{i}\right)$ is the corresponding price bid given by (15). If the corresponding score bid $s\left(c_{i}\right)=s\left(q\left(c_{i}\right), p\left(c_{i}\right)\right)$ is decreasing in types, then we have $\left(q\left(c_{i}\right), p\left(c_{i}\right)\right)$ constitutes a symmetric pure strategy equilibrium. Otherwise, there is no symmetric pure strategy equilibrium.

\section{An application to the ratio score}

As an application, we next show that with the ratio form score function $s=s(q, p)=\frac{q}{p}$, there might be no symmetric pure strategy equilibrium for the case of an all-pay quality bid, i.e. $\rho=1$. The equilibrium analysis in this application applies to a score function of $s=s(q, p)=\frac{q^{\mu}}{p^{\tau}}$ with $\mu, \tau>0$. 
In this case, (17) is written as

$$
\varsigma^{\prime}(q)=\frac{p}{q} \frac{\left[1-F\left(c_{i}\right)\right]^{N-1}}{c_{i}} .
$$

Given (15), we have

$$
\begin{aligned}
\varsigma^{\prime}\left(q\left(c_{i}\right)\right) & =\frac{1}{q\left(c_{i}\right)} \frac{\int_{c_{i}}^{\bar{c}} \varsigma(q(t)) d t+c_{i} \varsigma\left(q\left(c_{i}\right)\right)}{c_{i}} \\
\text { i.e., } c_{i} q\left(c_{i}\right) \varsigma^{\prime}\left(q\left(c_{i}\right)\right) & =\int_{c_{i}}^{\bar{c}} \varsigma(q(t)) d t+c_{i} \varsigma\left(q\left(c_{i}\right)\right) .
\end{aligned}
$$

Taking derivative wrt. $c_{i}$ both sides, we have

$$
q\left(c_{i}\right) \varsigma^{\prime}\left(q\left(c_{i}\right)\right)+c_{i} q\left(c_{i}\right) \varsigma^{\prime \prime}\left(q\left(c_{i}\right)\right) q^{\prime}\left(c_{i}\right)=0
$$

We thus have

$$
q^{\prime}\left(c_{i}\right)=-\frac{\varsigma^{\prime}\left(q\left(c_{i}\right)\right)}{c_{i} \varsigma^{\prime \prime}\left(q\left(c_{i}\right)\right)}<0
$$

with boundary condition $q(\bar{c})=0$.

Assume $\varsigma(q)=q^{\gamma}, \gamma>1$. We have

$$
\begin{aligned}
& (\gamma-1) \frac{q^{\prime}\left(c_{i}\right)}{q\left(c_{i}\right)}=-\frac{1}{c_{i}}<0 \\
& \text { i.e. } \frac{d \ln q\left(c_{i}\right)}{d c_{i}}=\frac{d\left[-\ln c_{i}\right]}{d c_{i}}
\end{aligned}
$$

with boundary condition $q(\bar{c})=0$.

We thus have

$$
\ln q\left(c_{i}\right)=-\frac{1}{\gamma-1} \ln c_{i}+A
$$

which gives

$$
q\left(c_{i}\right)=\left(\frac{1}{c_{i}}\right)^{\frac{1}{\gamma-1}} \exp (A) .
$$

Note we cannot have $q(\bar{c})=0$ if $\bar{c}$ is finite. Therefore, there exists no symmetric pure strategy equilibrium for this case.

However, if $\varsigma^{\prime}(0)>0$, then we indeed have a solution for $(19)$ with boundary condition $q(\bar{c})=0$, which can be identified as below.

(19) can be rewritten as

$$
\frac{d \varsigma^{\prime}\left(q\left(c_{i}\right)\right)}{d c_{i}}=\frac{d\left[-\ln c_{i}\right]}{d c_{i}}
$$

which entails

$$
\varsigma^{\prime}\left(q\left(c_{i}\right)\right)=\left(\frac{1}{c_{i}}\right) \exp (A) .
$$

Using the boundary condition of $q(\bar{c})=0$, we have

$$
\exp (A)=\bar{c} \varsigma^{\prime}(0)
$$


which leads to

$$
q\left(c_{i}\right)=\left(\varsigma^{\prime}\right)^{-1}\left(\frac{\bar{c} \varsigma^{\prime}(0)}{c_{i}}\right) .
$$

To confirm such identified $\left(q\left(c_{i}\right), p\left(c_{i}\right)\right)$ is indeed an equilibrium, we now only need to verify that $s\left(c_{i}\right)=$ $s\left(q\left(c_{i}\right), p\left(c_{i}\right)\right)=\frac{q\left(c_{i}\right)}{p\left(c_{i}\right)}$ is decreasing in types. Using (18) and (15) for $\rho=1$, we have

$$
\begin{aligned}
{\left[\ln s\left(c_{i}\right)\right]^{\prime} } & =\frac{q^{\prime}\left(c_{i}\right)}{q\left(c_{i}\right)}-\frac{p^{\prime}\left(c_{i}\right)}{p\left(c_{i}\right)}=\frac{1}{q\left(c_{i}\right)}\left[q^{\prime}\left(c_{i}\right)-\frac{q\left(c_{i}\right)}{p\left(c_{i}\right)} p^{\prime}\left(c_{i}\right)\right] \\
& =\frac{1}{q\left(c_{i}\right)}\left[q^{\prime}\left(c_{i}\right)-\left(\frac{c_{i} \varsigma^{\prime}\left(q\left(c_{i}\right)\right)}{\left[1-F\left(c_{i}\right)\right]^{N-1}}\right)^{-1}\left(\frac{c_{i} \varsigma^{\prime}\left(q\left(c_{i}\right)\right) q^{\prime}\left(c_{i}\right)}{\left[1-F\left(c_{i}\right)\right]^{N-1}}+(N-1) p\left(c_{i}\right) f\left(c_{i}\right)\right)\right] \\
& =-\frac{1}{q\left(c_{i}\right)}\left(\frac{c_{i} \varsigma^{\prime}\left(q\left(c_{i}\right)\right)}{\left[1-F\left(c_{i}\right)\right]^{N-1}}\right)^{-1}(N-1) p\left(c_{i}\right) f\left(c_{i}\right) \\
& <0
\end{aligned}
$$

which means $s^{\prime}\left(c_{i}\right)<0$.

An interesting observation is that with the ratio score, regardless of the number of players, all type distributions with the same support $[\underline{c}, \bar{c}]$ yield the same equilibrium quality bidding strategies $q\left(c_{i}\right), c_{i} \in[\underline{c}, \bar{c}]$. 


\section{References}

[1] Asker, John and Cantillon, Estelle. (2008). Properties of scoring auctions. Rand Journal of Economics, $39(1), 69-85$.

[2] Asker, John and Cantillon, Estelle. (2010). Procurement when price and quality matter. Rand Journal of Economics, 41(1), 1-34.

[3] Bajari, Patrick, Houghton, Stephanie and Tadelis Steven. (2014). Bidding for incomplete contract: an empirical analysis of adaptation costs. American Economic Review, 104(4), 1288-1319.

[4] Baye, M., D. Kovenock, and C. de Vries. (1989).The economics of all-pay, winner-take-all, contests. Working paper, Texas A\&M University, No. 89-21.

[5] Baye, M., D. Kovenock, and C. de Vries. (2005). Comparative analysis of litigation systems: an auction theoretic approach. The Economic Journal, 115, 583-601.

[6] Branco, Fernando. (1997). The design of multidimensional auctions. Rand Journal of Economics, 28(1), $63-81$.

[7] Che, Yeon-Koo. (1993). Design competition through multidimensional auctions. Rand Journal of Economics, 24(4), 668-680.

[8] Che, Yeon-Koo and Gale, Ian. (2003). Optimal design of research contests. American Economic Review, 93(3), 646-671.

[9] Hanazono, Makoto, Nakabayashi, Jun and Tsuruoka, Masanori. (2015). Procurement auctions with general price-quality evaluation. Working paper.

[10] Kaplan, T., Luski, I., Sela A., and Wettstein, D. (2002) All-pay auctions with variable rewards. Journal of Industrial Economics, 2002(4), 417-430.

[11] Myerson, Roger B. (1981). Optimal auction design. Mathematics of Operations Research, 6(1), 58-73.

[12] Nishimura, Takeshi. (2015). Optimal design of scoring auctions with multidimensional quality. Working paper.

[13] Ron Siegel. (2010). Asymmetric contests with conditional investments. American Economic Review, $100(5), 2230-2260$.

[14] Wang, Mingxi and Liu, Shulin. (2014). Equilibrium bids in practical multi-attribute auctions. Economics Letters, 123, 352-355. 